\title{
Effect of insulin-like growth factor-1 and hyaluronic acid in experimentally produced osteochondral defects in rats
}

\author{
Celil Alemdar, İstemi Yücel ${ }^{1}$, Barış Erbil ${ }^{2}$, Havva Erdem ${ }^{3}$ Ramazan Atiç, Emin Özkul
}

\begin{abstract}
Background: The common purpose of almost all methods used to treat the osteochondral injuries is to produce a normal cartilage matrix. However current methods are not sufficient to provide a normal cartilage matrix. For that reason, researchers have studied to increase the effectiveness of this methods using chondrogenic and chondroprotective molecules in recent experimental studies. Insulin-like growth factor-1 (IGF-1) and hyaluronic acid (HA) are two important agents used in this field. This study compared the effects of IGF-1 and HA in an experimental osteochondral defect in rat femora.

Materials and Methods: The rats were divided into three groups ( $n=15$ per group) as follows: The IGF-1 group, HA group, and control group. An osteochondral defect of a diameter of $1.5 \mathrm{~mm}$ and a depth of $2 \mathrm{~mm}$ was created on the patellar joint side of femoral condyles. The IGF-1 group received an absorbable gelatin sponge soaked with $15 \mu \mathrm{g} / 15 \mu \mathrm{l}$ of IGF-1, and the HA group received an absorbable gelatin sponge soaked with $80 \mu \mathrm{g} \mathrm{HA}$. The control group received only an absorbable gelatin sponge. Rats were sacrificed at the $6^{\text {th }}$ week, and the femur condyles were evaluated histologically.

Results: According to the total Mankin scale, there was a statistically significant difference between IGF-1 and HA groups and between IGF-1 and control groups. There was also a significant statistical difference between HA and control groups.

Conclusion: It was shown histopathologically that IGF-1 is an effective molecule for osteochondral lesions. Although it is weaker than IGF-1, HA also strengthened the repair tissue.
\end{abstract}

Key words: Cartilage, hyaluronic acid, insulin-like growth factor-1, osteochondral defect MeSH terms: Hyaluronic acid, cartilage, IGF-1, sports medicine, rats

\section{INTRODUCTION}

$\mathrm{O}$ steochondral lesions, mostly seen in the young population and men, are most commonly localized in the knee, elbow, and foot. ${ }^{1}$ These lesions, which are usually due to trauma, are significant clinical issues because of the low ability to recover and leading

Department of Orthopaedics, Dicle University Medical Faculty, Diyarbakir, ${ }^{1}$ Department of Orthopaedics, Düzce University Medical Faculty, Düzce, ${ }^{2}$ Department of Orthopaedics, Tekirdağ State Hospital, Tekirdağ,

${ }^{3}$ Department of Pathology, Ordu University Medical Faculty, Ordu, Turkey

Address for correspondence: Prof. Celil Alemdar,

Department of Orthopaedics, Dicle University Medical Faculty,

21280, Diyarbakir, Turkey.

E-mail: celilalemdar@mynet.com

\begin{tabular}{|l|l|}
\hline \multicolumn{2}{|c|}{ Access this article online } \\
\hline Quick Response Code: & Website: \\
\hline & www.ijoonline.com \\
\cline { 2 - 2 } & \\
\hline
\end{tabular}

to osteoarthritis. ${ }^{2}$ Many surgical approaches are used to treat these lesions. Periosteal or osteochondral grafting to strengthen repair tissue, bone marrow stimulation methods (microfracture, abrasion arthroplasty, and drilling), and cell-based approaches that have become popular in recent years (autologous chondrocyte transplantation and supportive matrix applications) are employed in its surgical treatment. ${ }^{3,4}$

Unlike partial cartilage defects, osteochondral defects can be repaired through the migration of mesenchymal stem cells from subchondral bone. However, since the ability

This is an open access article distributed under the terms of the Creative Commons Attribution-NonCommercial-ShareAlike 3.0 License, which allows others to remix, tweak, and build upon the work non-commercially, as long as the author is credited and the new creations are licensed under the identical terms.

For reprints contact: reprints@medknow.com

How to cite this article: Alemdar C, Yücel İ, Erbil B, Erdem H, Atiç R, Özkul E. Effect of insulin-like growth factor-1 and hyaluronic acid in experimentally produced osteochondral defects in rats. Indian J Orthop 2016;50:414-20. 
of mesenchymal cells to differentiate into chondral cells is low, the newly-formed cartilage tissue does not completely reflect the basic characteristics of normal hyaline cartilage. ${ }^{2}$ This kind of recovery is called fibrocartilage recovery due to the fibrous content. The common purpose of almost all studies that have been conducted on osteochondral lesions is to increase the recovery potential of osteochondral lesions where mesenchymal cells play a key role and to enhance the differentiation ability of stem cells. In this way, organized chondral tissue production can be achieved with a cellular content that can imitate normal hyaline cartilage and an extracellular matrix structure.

Insulin-like growth factor (IGF) is a peptide hormone synthesized mainly in the liver, which plays an active role in several metabolic events within the body and displays anabolic activity through local and systemic pathways. IGF-1 also activates cell proliferation and differentiation through receptor which has a higher affinity for IGF-1. ${ }^{5}$ It exerts this effect through the mesenchymal stem cells. This molecule strengthens repair tissue by increasing the synthesis of DNA, proteoglycan, collagen, and other extracellular matrix proteins, and prevents pain and joint degeneration by reducing the chronic synovial inflammation and the catabolism of extracellular matrix proteins. ${ }^{6}$ In this respect, it is important for the recovery of osteochondral defects.

Hyaluronic acid (HA) is a glycosaminoglycan polysaccharide, which has important roles in the joints. ${ }^{7}$ Mostly, it is used for viscosupplementation to reduce pain in diseases with diffuse cartilage damage such as osteoarthritis and to increase the functional capacity. ${ }^{8}$ It displays a protective effect on chondrocytes and the extracellular matrix by reducing inflammation and the enzymes with catabolic effect, whereas it has a chondroproliferative effect through the stimulation of the stem cell proliferation and differentiation and the synthesis of matrix proteins. ${ }^{9}$ In addition, it increases angiogenesis and thereby allows for much faster tissue repair. ${ }^{10}$ In order to benefit from these effects, the cartilage engineering studies place emphasis on it for scaffold manufacturing. ${ }^{11,12}$

IGF and HA are two popular molecules that have been researched in many studies. ${ }^{5,11}$ On the advancement of cartilage engineering, the efficacy of these molecules in osteochondral defects has been an attractive area of research. This study compared the efficacy of IGF-1 and HA applications in rats with experimentally created osteochondral defects.

\section{Materials and Methods}

Forty-five adult male Wistar Albino rats were used in this study according to all institutional and national guidelines for the care and use of laboratory animals. The rats had a weight of 220-275 g and were aged 5-7 months. The subjects were divided into three groups of 15 rats each, which was enough for statistical assessment. The first group was identified as the IGF-1 group, second group as the HA group, and the third group as the control group.

\section{Operative procedure}

An arthrotomy was performed on the knees of the rats by medial parapatellar incision. The patella was rolled laterally, and the anterior joint surface of the distal femur was opened. A cylindrical full-thickness osteochondral defect with a diameter of $1.5 \mathrm{~mm}$ and a depth of $2 \mathrm{~mm}$ was formed on the femoral joint surface of the patellofemoral joint with a drill. An absorbable gelatin sponge with a diameter of $3 \mathrm{~mm}$ and a thickness of $2 \mathrm{~mm}$ (Spongostan Standart, Ferrosan Medical, Soeborg, Denmark) was packed into the defect [Figure 1]. The injection into the sponge was made from different zones with fine-needle injectors at a dose of $15 \mu \mathrm{g} / 15 \mu \mathrm{l}$ IGF-1 (R IGF-1, Sigma-Aldrich, Missouri, USA) in the first group (the IGF-1 group) and $80 \mu \mathrm{g}$ HA (Ostenil $20 \mathrm{mg}$, Bio-Gen Pharmaceuticals, Ankara, Turkey) in the second group (the HA group) (the IGF-1 and HA doses were determined by considering the maximum volume that the defect could take together with the absorbable sponge). Only the absorbable gelatin sponge was packed in the third group (the control group).

\section{Morphological and histopathological assessments}

One rat from the first group and two rats from the third group were excluded from the study due to death from an unknown cause and one rat from the third group was excluded due to an acute infection. The distal femurs of the remaining rats which were killed by injecting a lethal dose of phenobarbital were removed at the end of week 6 .

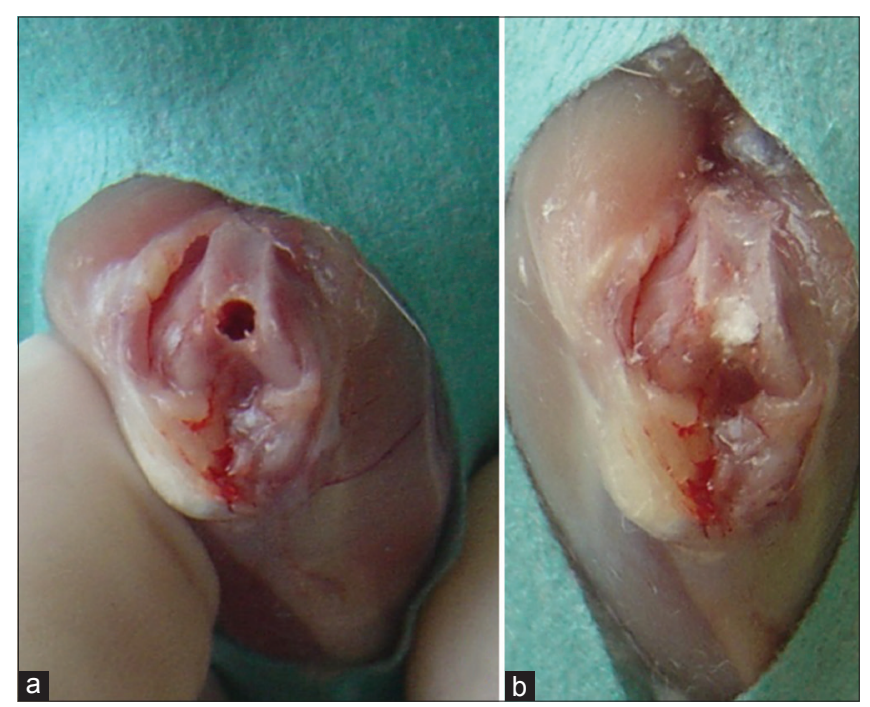

Figure 1: (a) Distal femoral joint surface with created osteochondral defect. (b) The view of rat knee administered insulin-like growth factor-1-soaked absorbable gelatin sponge 
In total, 14, 15, and 12 samples were obtained in the first, second, and third groups, respectively.

The obtained tissue samples were fixed in a $10 \%$ formalin ( $\mathrm{pH}=7.0$ ) solution prepared within a phosphate buffer and then decalcified at the room temperature in DeCastro (30 ml 70\% concentrated nitric acid, $50 \mathrm{~g}$ chloral hydrate, $300 \mathrm{ml}$ absolute ethanol) solution in a controlled manner. The samples were embedded in paraffin through routine tissue followup after decalcification and a series of cross-sections of the sagittal plane were taken with a Rotary Microtome (Shandon MB35 Premier) and placed on the gelatin-covered slides from the paraffin blocks at a thickness of $4 \mu \mathrm{m}$ by skipping once in 2-3 times to find the defect. The obtained histological cross-sections were stained with hematoxylin and eosin and the Safranin-O technique. All cross-sections were evaluated by people specialized in their field in a double-blind manner under a light microscope. The tissue samples representing the lesion were examined for the cartilage structure of the repair tissue, cellular arrangement, matrix staining with Safranin-O, and the joint surface uniformity. The results were evaluated based on the Mankin scale. ${ }^{13}$

\section{Statistical analysis}

Statistical analysis was performed using SPSS 13.0 (SPSS, Chicago, Illinois, USA). The one-sample KolmogorovSmirnov test was used to evaluate the distribution of the groups. Since the groups were not normally distributed, nonparametric Kruskal-Wallis test and Mann-Whitney U-test were used to make statistical assessments between the groups. A $P<0.05$ was considered statistically significant.

\section{Results}

The morphological assessment was conducted by considering the recovery of the osteochondral defect [Figure 2]. The defect was covered better in the IGF-1 and HA groups compared to the control group. The comparison between the IGF-1 and HA groups revealed that the defect was covered better in the IGF-1 group.

The preparations were evaluated for the cartilage structure, cellular abnormality, matrix staining, and tidemark continuity and were photographed [Figures 3 and 4]. In the IGF-1 group, the cartilage structure was $1.21 \pm 0.58$, cellular abnormality was $1.29 \pm 0.47$, matrix staining was $2.14 \pm 0.95$, and tidemark continuity was $0.57 \pm 0.51$. In the HA group, the cartilage structure was $1.93 \pm 0.88$, cellular abnormality was $1.73 \pm 0.70$, matrix staining was $3.06 \pm 0.59$, and tidemark continuity was $0.67 \pm 0.49$. In the control group, the cartilage structure was $3.50 \pm 0.57$, cellular abnormality was $2.33 \pm 0.78$, matrix staining was $3.00 \pm 0.74$, and tidemark continuity was $1.00 \pm 0.00$. The IGF-1 group was found to have lower values compared to the total Mankin scale and also to have lower mean cartilage structure, cellular abnormality, matrix staining, and tidemark continuity values compared to both HA and control groups. The minimum and maximum values of the groups' histological examinations based on Mankin Classification are presented in Table 1.

There was a statistically significant difference in cartilage structure and matrix staining between the IGF-1 and HA groups ( $P=0.005, P=0.010$; respectively); whereas no statistically significant difference was found in cellular

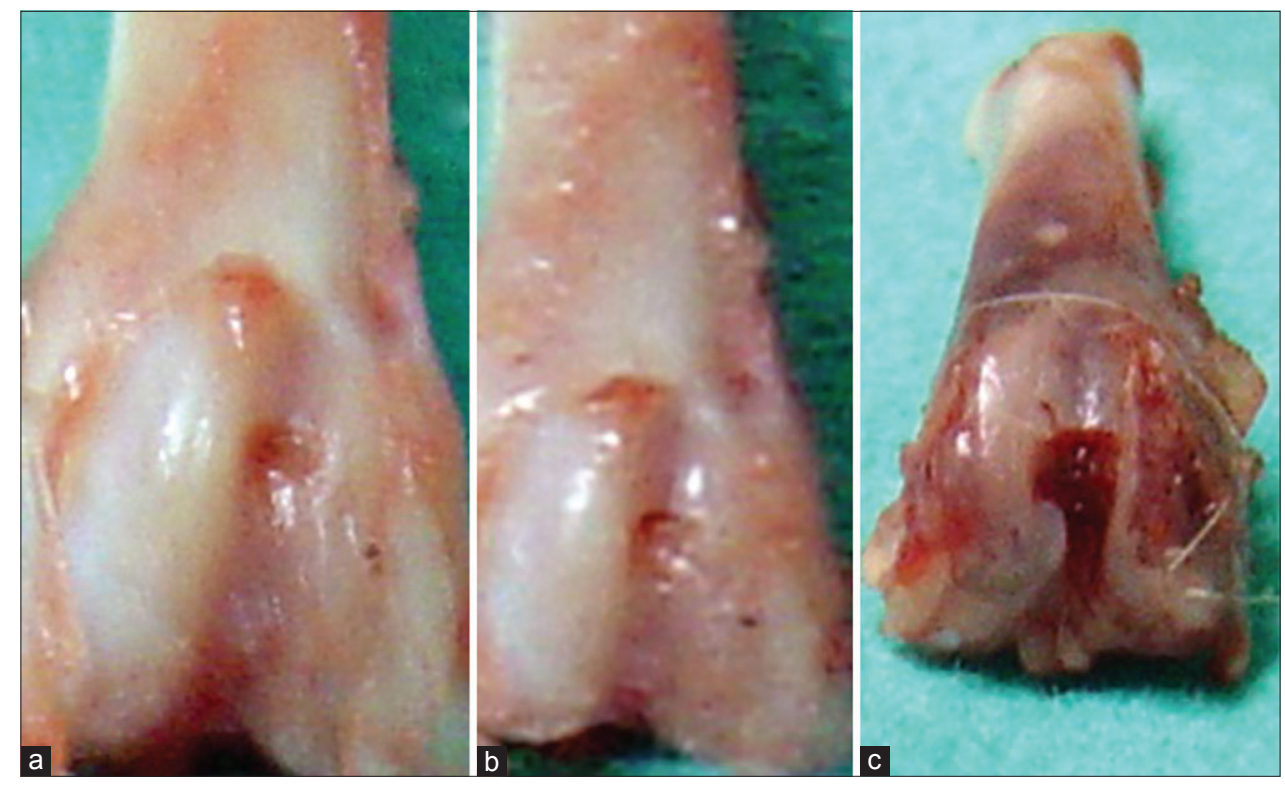

Figure 2: The macroscopic view of pieces obtained at postoperative week 6: (a) from the insulin-like growth factor-1 group, (b) from the hyaluronic acid group, and (c) from the control group showing that the defect was covered better in IGF-1 and HA groups 


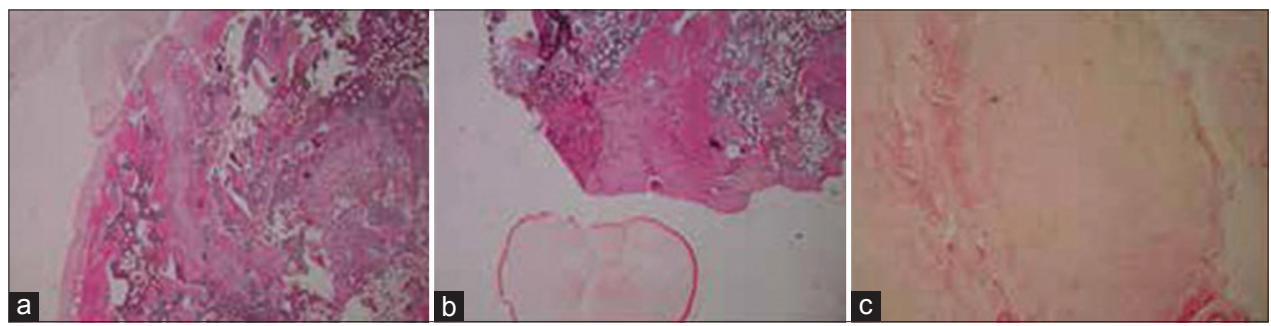

Figure 3: Insulin-like growth factor-1 group (a) was found to have less fissure development and pannus and better cellularity compared to (b) the hyaluronic acid group (c) and the control group $(\mathrm{H}$ and $\mathrm{E}, \times 40)$
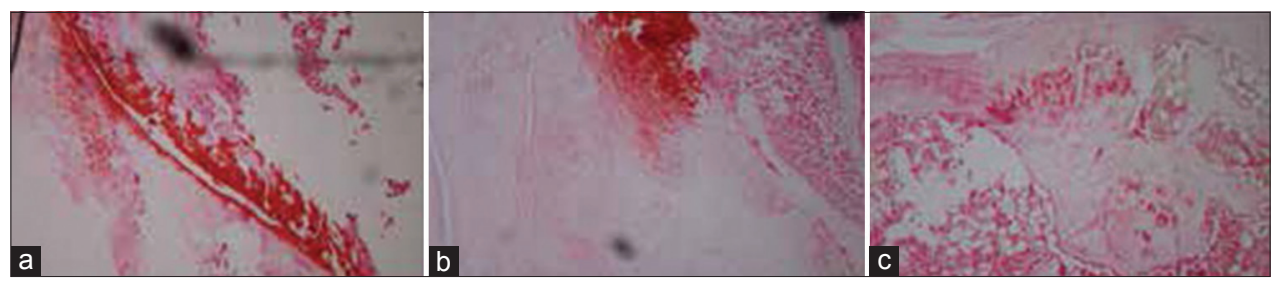

Figure 4: Insulin-like growth factor-1 group (a) was found to have better matrix staining compared to (b) the hyaluronic acid group (c) and the control group (Safranine-O, $\times 100$ )

Table 1: The minimum and maximum values of the group's histological examinations based on the Mankin classification (the low value is good healing, the high value is poor healing)

\begin{tabular}{lcccc}
\hline Cartilage & $\begin{array}{c}\text { Cellular } \\
\text { structure }\end{array}$ & $\begin{array}{c}\text { Matrix } \\
\text { abnormality }\end{array}$ & $\begin{array}{c}\text { Tidemark } \\
\text { staining }\end{array}$ & Continuity \\
\hline IGF-1 group & $1-3$ & $1-2$ & $1-4$ & $0-1$ \\
HA group & $1-3$ & $1-3$ & $2-4$ & $0-1$ \\
Control group & $1-6$ & $1-3$ & $2-4$ & $1-1$ \\
\hline
\end{tabular}

IGF=Insulin-like growth factor, HA=Hyaluronic acid

abnormality and tidemark continuity $(P=0.057, P=0.683$; respectively). There was a statistically significant difference in cartilage structure, cellular abnormality, and matrix staining between the IGF-1 and control groups $(P=0.001$, $P=0.001$, and $P=0.031$, respectively), whereas no statistically significant difference was found in tidemark continuity $(P=0.067)$. There was a statistically significant difference in cartilage structure between the HA and control groups $(P=0.012)$, whereas no statistically significant difference was found in cellular abnormality, matrix staining, and tidemark continuity $(P=0.067, P=0.683, P=0.152$; respectively). There was a statistically significant difference between the IGF-1 and HA groups, the IGF-1 and control groups and the HA and control groups in terms of total Mankin scale $(P=0.001, P=0.001$, and $P=0.007)$.

\section{Discussion}

The common purpose of almost all methods used to treat the osteochondral injuries is to make the mesenchymal stem cells migrate to the defect area and then differentiate, proliferate, and produce a normal cartilage matrix. ${ }^{14}$ Cartilage engineering has made a significant progress in this field and developed many products for use in osteochondral injuries in recent years. In particular, the scaffolds containing chondrocytes are commonly used. ${ }^{15,16}$ These scaffolds contain chondrocytes produced in culture media and function as normal hyaline cartilage when applied to the osteochondral defects. However, they are effective to the extent that they imitate the normal hyaline cartilage. The agents with chondrogenic and chondroprotective effects are mainly emphasized in order to increase the similarity of the defect to the natural cartilage. IGF-1 and $\mathrm{HA}$, from the prominent agents of this matter, were compared in this study.

IGF-1 plays a great role in normal development and healthy functioning of the musculoskeletal system. ${ }^{16}$ It induces mesenchymal cell migration, proliferation, and differentiation. It also increases the biosynthesis of mRNA and matrix proteins. ${ }^{17}$ These effects have made IGF-1 become one of the important actors of the researches on osteochondral injury. Kim et al. ${ }^{18}$ conducted a study using a double-layer composite hydrogel and evaluated the efficacy of IGF-1 and the efficacy of transforming growth factor- $\beta 3$ (TGF- $\beta 3$ ) in rabbit osteochondral defects. In the histopathological examination, the cartilage development was found significantly better in the groups who received IGF-1 and IGF-1 + TGF- $\beta 3$ compared to the control group. The authors also reported that IGF-1 exerted its positive effect independently of TGF- $\beta 3$, and there was no synergistic action between these two molecules. Orth et al. ${ }^{19}$ transinfected the chondrocytes from rabbit hips and knees with IGF-1 and fibroblast growth factor- 2 and applied these transinfected chondrocytes on the rabbit knees with created osteochondral defects in their study. Examination at 3 weeks after the surgery, it was found that there were less fibrocartilage development, significantly better proteoglycan and cartilage repair, and less degenerative modifications in the subjects who received gene expression-induced chondrocytes. 
Alemdar, et al.: IGF-1 and HA in osteochondral lesions

In another study, Leng et al. ${ }^{20}$ (2012) reported that they achieved greater positive outcomes in the morphological, microscopic, electron microscopic, and magnetic resonance imaging examinations in goat knees with created large osteochondral defects upon using IGF-1. Furthermore, the release of anabolic cytokine was higher in the IGF-1 group. Singh et al. ${ }^{5}$ (2007) also demonstrated that the use of IGF-1 provided a significant contribution to cartilage recovery in their study with rabbits.

Verwilghen et al. ${ }^{21}$ (2009) evaluated the efficacy of IGF-1 on horses. Their study investigated the reaction of IGF-1 levels against trauma in the plasma of the horses after an osteochondral defect. For this purpose, horses with osteochondral defects and healthy control horses were compared. The plasma levels of IGF-1 were higher in horses with osteochondral defects. They suggested that this difference was associated with the repair of the osteochondral defect, and they indicated the importance of this IGF-1 for the cartilage repair.

Another molecule used in many studies on cartilage tissue is HA. HA regulates the viscoelasticity of synovial fluid. ${ }^{22}$ In addition, it has many important functions such as creating a lubricant effect, increasing the synthesis of matrix proteins, and protecting joints from catabolic agents. ${ }^{23}$ Mostly used in osteoarthritis; HA has been also studied in osteochondral defects in recent years. Park et al. ${ }^{24}$ (2014) conducted a study in tissue engineering and evaluated the proliferation, migration, and extracellular matrix production of the human-origin osteoblasts and chondrocytes in culture medium using type I collagen hydrogel and HA hydrogel. The researchers presented that the osteoblasts were better produced in type I collagen, whereas the chondrocytes were better produced in HA. Based on this result, it has been reported that it is possible to successfully prepare three-dimensional osteochondral tissue grafts by using type I collagen matrix for the osseous part and HA matrix for the chondral layer, as it is composed of two separate components and to use such grafts in osteochondral lesions. Suwannaloet et al. ${ }^{25}$ (2012) compared the efficacy of saline, HA, and diacerein in rabbits with created chondral defects in the knees. The authors reported that HA, which was administered after subchondral drilling, increased especially collagen production and chondrocyte stimulation, and there was an increased effect of HA in the group that received diacerein additionally. Kon et al. ${ }^{9}$ created an osteochondral defect in medial and lateral femoral condyles of goats and reported better outcomes and increased regeneration ability due to HA administration in the groups that had drilling in the histopathological and morphological assessment after 6 months. Kim et al. ${ }^{26}$ (2014) reported that both HA and MSC (mesenchymal stem cell) applications increased the regeneration ability in the rabbits with created osteochondral defects; however, the outcomes were better if both were used in combination.

The effect of HA on osteochondral defects has been also evaluated in clinical trials. Doral et al. ${ }^{27}$ (2012) reported the outcomes of 57 patients with osteochondral defects of the talus. The patients who were treated arthroscopically and had perfusion and debridement via the microfracture method were evaluated in two groups: Patients who were administered and those that were not administered injection of HA. The postoperative assessment was performed in terms of functional scores/outcome and pain levels. The postoperative scores were found significantly higher than the preoperative scores in both groups. When the postoperative scores of both groups were compared, the scores were better in the group that received HA. The authors reported that the microfracture treatment was an effective approach, and the treatment efficacy would increase if HA was added.

There are a limited number of studies comparing IGF-1 and HA in articular cartilage. One of these is the study by Liu et al. (2011). ${ }^{28}$ This study was conducted on the rabbit temporomandibular joints with created osteoarthritis. The histopathological examination at postoperative months 12 and 24 revealed no significant difference between the control, HA, and IGF-1 groups. This result is contrary to many studies creating the osteochondral defect model reported in the literature. However, it is different to conduct the study on the temporomandibular joint where osteoarthritis rarely develops in an osteoarthritis model and in practice. The authors also reported that the group receiving the HA and IGF-1 combination was significantly superior compared to other groups, and the co-administration of these two molecules would provide a successful outcome in osteoarthritis.

In this study, the results of the IGF-1 and HA groups were superior to the control group based on the total Mankin scale. In addition, the results of the IGF-1 group were superior to the HA group. Most of the studies on osteochondral defects support the current findings. In that sense, the current findings are consistent with the literature. As distinct from this study, the demonstration that the co-administration of these two agents, one of which is mainly chondroblastic and the other is chondroprotective, results in a synergistic action are important, as well.

HA is used in chondral tissues produced for osteochondral defects. There are several clinical studies related to these products which contain HA. ${ }^{29,30}$ On the contrary, there are only experimental studies related to IGF-1 in literature. ${ }^{17,19}$ Clinical trials are not yet available. If the effectiveness of IGF-1 is demonstrated with similar studies, we think this molecule will be used in clinical applications in the coming 
years. That is, IGF-1 may be added into scaffold used for osteochondral defects.

The limitations of our study are the short followup.

\section{Conclusion}

It has been histopathologically presented in this experimental study that IGF-1 is an effective molecule in osteochondral lesions. Although less potent than IGF-1, HA strengthens the repair tissue, as well. The studies combining these two molecules in order to create a synergistic action will provide positive contributions to the recovery process of osteochondral injuries.

\section{Financial support and sponsorship}

Nil.

\section{Conflicts of interest}

There are no conflicts of interest.

\section{References}

1. Mei-Dan O, Carmont MR, Laver L, Mann G, Maffulli N, Nyska M. Platelet-rich plasma or hyaluronate in the management of osteochondral lesions of the talus. Am J Sports Med 2012;40:534-41.

2. Bail H, Klein P, Kolbeck S, Krummrey G, Weiler A, Schmidmaier G, et al. Systemic application of growth hormone enhances the early healing phase of osteochondral defects - A preliminary study in micropigs. Bone 2003;32:457-67.

3. Oldinski RA, Ruckh TT, Staiger MP, Popat KC, James SP. Dynamic mechanical analysis and biomineralization of hyaluronan-polyethylene copolymers for potential use in osteochondral defect repair. Acta Biomater 2011;7:1184-91.

4. Madry H, Orth P, Kaul G, Zurakowski D, Menger MD, Kohn D, et al. Acceleration of articular cartilage repair by combined gene transfer of human insulin-like growth factor I and fibroblast growth factor-2 in vivo. Arch Orthop Trauma Surg 2010;130:1311-22.

5. Singh NK, Singh GR, Amarpal, Kinjavdekar P, Sharma AK, Mohanty TR, et al. Articular cartilage repair with autografting under the influence of insulin-like growth factor-1 in rabbits. J Vet Med A Physiol Pathol Clin Med 2007;54:210-8.

6. Fortier LA, Barker JU, Strauss EJ, McCarrel TM, Cole BJ. The role of growth factors in cartilage repair. Clin Orthop Relat Res 2011;469:2706-15.

7. Elmalı N, Kaygusuz MA, Özen S, Baysal Ö, İnan M, Karakaplan M, et al. The healing effect of intraarticular hyaluronic acid injection on osteoarthritic knee: (A study on rabbit knees). Acta Orthop Traumatol Turc 1999;33:211-5.

8. Bert JM, Waddell DD. Viscosupplementation with hylan g-f 20 in patients with osteoarthrosis of the knee. Ther Adv Musculoskelet Dis 2010;2:127-32.

9. Kon E, Filardo G, Robinson D, Eisman JA, Levy A, Zaslav K, et al. Osteochondral regeneration using a novel aragonite-hyaluronate bi-phasic scaffold in a goat model. Knee Surg Sports Traumatol Arthrosc 2014;22:1452-64.
10. Tekin AC, Esenyel CZ, Cakar M, Esenyel M, Ozcan Y, Saygili MS. Hyalonect in the treatment of pseudarthrosis. Acta Orthop Traumatol Turc 2013;47:379-86.

11. Lebourg M, Martínez-Díaz S, García-Giralt N, Torres-Claramunt R, Gómez-Tejedor JA, Ribelles JL, et al. Cell-free cartilage engineering approach using hyaluronic acid-polycaprolactone scaffolds: A study in vivo. J Biomater Appl 2014;28:1304-15.

12. Unterman SA, Gibson M, Lee JH, Crist J, Chansakul T, Yang EC, et al. Hyaluronic acid-binding scaffold for articular cartilage repair. Tissue Eng Part A 2012;18:2497-506.

13. Mankin HJ, Dorfman H, Lippiello L, Zarins A. Biochemical and metabolic abnormalities in articular cartilage from osteo-arthritic human hips. II. Correlation of morphology with biochemical and metabolic data. J Bone Joint Surg Am 1971;53:523-37.

14. Miller RE, Grodzinsky AJ, Vanderploeg EJ, Lee C, Ferris DJ, Barrett MF, et al. Effect of self-assembling peptide, chondrogenic factors, and bone marrow-derived stromal cells on osteochondral repair. Osteoarthritis Cartilage 2010;18:1608-19.

15. Gelse K, Mühle C, Knaup K, Swoboda B, Wiesener M, Hennig F, et al. Chondrogenic differentiation of growth factor-stimulated precursor cells in cartilage repair tissue is associated with increased HIF-1alpha activity. Osteoarthritis Cartilage 2008; 16:1457-65.

16. Kim HJ, Lee WJ. Insulin-like growth factor-I induces androgen receptor activation in differentiating $\mathrm{C} 2 \mathrm{C} 12$ skeletal muscle cells. Mol Cells 2009;28:189-94.

17. Karna E, Miltyk W, Surazynski A, Palka JA. Protective effect of hyaluronic acid on interleukin-1-induced deregulation of beta1-integrin and insulin-like growth factor-I receptor signaling and collagen biosynthesis in cultured human chondrocytes. Mol Cell Biochem 2008;308:57-64.

18. Kim K, Lam J, Lu S, Spicer PP, Lueckgen A, Tabata Y, et al. Osteochondral tissue regeneration using a bilayered composite hydrogel with modulating dual growth factor release kinetics in a rabbit model. J Control Release 2013;168:166-78.

19. Orth P, Kaul G, Cucchiarini M, Zurakowski D, Menger MD, Kohn D, et al. Transplanted articular chondrocytes co-overexpressing IGF-I and FGF-2 stimulate cartilage repair in vivo. Knee Surg Sports Traumatol Arthrosc 2011;19:2119-30.

20. Leng $P$, Ding CR, Zhang HN, Wang YZ. Reconstruct large osteochondral defects of the knee with hIGF-1 gene enhanced Mosaicplasty. Knee 2012;19:804-11.

21. Verwilghen DR, Vanderheyden L, Franck T, Busoni V, Enzerink E, Gangl M, et al. Variations of plasmatic concentrations of Insulin-like Growth Factor-I in post-pubescent horses affected with developmental osteochondral lesions. Vet Res Commun 2009;33:701-9.

22. Wang CT, Lin J, Chang CJ, Lin YT, Hou SM. Therapeutic effects of hyaluronic acid on osteoarthritis of the knee. A meta-analysis of randomized controlled trials. J Bone Joint Surg Am 2004;86-A: 538-45.

23. Akman Ş, Şen C, Gögüş A, Demirhan M, Kılıçoğlu Ö. The efficacy of intraarticular sodium hyaluronate injection following arthroscopic debridement in the treatment of gonarthrosis. Acta Orthop Traumatol Turc 2001;35:107-10.

24. Park JY, Choi JC, Shim JH, Lee JS, Park H, Kim SW, et al. A comparative study on collagen type I and hyaluronic acid dependent cell behavior for osteochondral tissue bioprinting. Biofabrication 2014;6:035004.

25. Suwannaloet W, Laupattarakasem W, Sukon P, Ong-Chai S, 
Alemdar, et al.: IGF-1 and HA in osteochondral lesions

Laupattarakasem P. Combined effect of subchondral drilling and hyaluronic acid with/without diacerein in full-thickness articular cartilage lesion in rabbits. Scientific World Journal 2012;2012:310745.

26. Kim SS, Kang MS, Lee KY, Lee MJ, Wang L, Kim HJ. Therapeutic effects of mesenchymal stem cells and hyaluronic Acid injection on osteochondral defects in rabbits' knees. Knee Surg Relat Res 2012;24:164-72.

27. Doral MN, Bilge O, Batmaz G, Donmez G, Turhan E, Demirel M, et al. Treatment of osteochondral lesions of the talus with microfracture technique and postoperative hyaluronan injection. Knee Surg Sports Traumatol Arthrosc 2012;20:1398-403.

28. Liu XW, Hu J, Man C, Zhang B, Ma YQ, Zhu SS. Insulin-like growth factor-1 suspended in hyaluronan improves cartilage and subchondral cancellous bone repair in osteoarthritis of temporomandibular joint. Int J Oral Maxillofac Surg 2011;40:184-90.

29. Ossendorf C, Kaps C, Kreuz PC, Burmester GR, Sittinger M, Erggelet C. Treatment of posttraumatic and focal osteoarthritic cartilage defects of the knee with autologous polymer-based three-dimensional chondrocyte grafts: 2-year clinical results. Arthritis Res Ther 2007;9:1-11.

30. Kreuz PC, Müller S, Ossendorf C, Kaps C, Erggelet C. Treatment of focal degenerative cartilage defects with polymer-based autologous chondrocyte grafts: Four-year clinical results. Arthritis Res Ther 2009;11:R33. 